\title{
Simulation on the effects of various factors on the motion of ultrasonic cavitation bubble
}

\author{
Wanxin Qu, Yuanhua Xie*, Yang Shen, Jin Han, Meiyan You, Tong Zhu \\ School of Mechanical Engineering and Automation, Northeastern University, Shenyang 110004, \\ China
}

Email: yhxie@mail.neu.edu.cn

\begin{abstract}
This paper aims to disclose the exact effects of various factors on the motion of ultrasonic cavitation bubble. For this purpose, this paper modifies the cavitation bubble dynamics model in light of liquid compressibility, liquid viscosity, water evaporation and vapor condensation. Based on the model, a dynamic equation was created for the bubble, and applied for Matlab simulation of bubble motion. The bubble motion was characterized by such parameters as radius, temperature, pressure, internal energy and the number of vapor molecules. The simulation attempts to reveal how the bubble motion is affected by the initial bubble radius, the ultrasonic frequency and the ultrasonic amplitude. Through the simulation, it is discovered that the change of the initial radius had a little impact on the bubble temperature and bubble pressure. However, the radius variation exerted an obvious influence on the other motion parameters. These parameters shared a similar change pattern. For ultrasonic frequency, the change in frequency had a limited effect on the bubble temperature and bubble pressure, but a significant impact on the other motion parameters. These parameters varied in different ranges. In addition, the ultrasonic amplitude had a rather prominent impact on all motion parameters of the cavitation bubble. The research findings provide important insight into the dynamic features of ultrasonic cavitation under ultrasonic waves.
\end{abstract}

Keywords: Ultrasonic Cavitation, Cavitation Bubble, Bubble Radius, Ultrasonic Frequency, Ultrasonic Amplitude.

\section{INTRODUCTION}

Ultrasonic cavitation often occurs in liquids under sufficiently strong ultrasonic waves. Due to the interaction between the positive and negative components, the micro bubble nucleus in the liquid will expand, shrink, collapse and even vibrate after the collapse. In the phase of collapse, the bubble usually exhibits high temperature, high pressure, shock wave and micro jet [1].

Many equations have been developed to fit the motion of the cavitation bubble. Inspired by the Rayleigh equation, Plesset et al. [2] put forward Rayleigh-Plesset (RP) equation and Rayleigh-Plesset Noltingk Neppiras and Poritsky (RPNNP) equation after exploring the dynamic process of cavitation bubble in fluid mechanics. Noltingk, Neppiras and Gilmore [3,4] derived another dynamic equation of ultrasonic cavitation bubble from the Rayleigh equation, considering various influencing factors. An et al. [5] investigated how chemical reaction and many other factors affect acoustic cavitation bubble. Wang et al. [6] conducted a qualitative analysis on the effects of liquid and sound field parameters on the maximum temperature and pressure of the bubble, but did not carry out a quantitative analysis. $\mathrm{Xu}$ et al. [7] modified the RPNNP equation to disclose the influence of the following factors on the motion of cavitation bubble when the ultrasonic wave propagates in water, namely, ultrasonic frequency, ultrasonic power, and the initial equilibrium radius of the bubble. They also studied the influence of sound pressure amplitude and main liquid phase temperature on bubble temperature and pressure in the collapse phase.

In general, the above equations fail to simulate the exact motion of cavitation bubble, due to the neglecting of such factors as liquid compressibility, water evaporation and vapor condensation. To solve the problem, this paper creates a dynamic model of ultrasonic cavitation in light of liquid compressibility, liquid viscosity, water evaporation and vapor condensation, and introduces the model to the motion simulation of cavitation bubble. The goal is to disclose how the bubble motion is affected by the initial bubble radius, the ultrasonic frequency and the ultrasonic amplitude.

\section{DYNAMIC MODEL OF ULTRASONIC CAVITATION}

The Yasui Kyuichi equations were adopted to depict the exact dynamic behaviour of ultrasonic cavitation bubble. The 
equations take account of the thermal boundary layer in the inner and outer boundaries of the bubble [8-11]. Considering the effect of the external boundary pressure gradient on the radial velocity of the bubble, the bubble radius equation was modified as:

$\left(1-\frac{\dot{R}}{c}+\frac{\dot{m}}{c \rho}\right) R \ddot{R}+\frac{3}{2} \dot{R}^{2}\left(1-\frac{\dot{R}}{3 c}+\frac{2 \dot{m}}{3 c \rho}\right)=$

$\frac{1}{\rho}\left(1+\frac{\dot{R}}{c}\right)\left[P_{B}-P_{s}\left(t+\frac{R}{c}\right)-P_{0}\right]+\frac{\ddot{m} R}{\rho}\left(1-\frac{\dot{R}}{c}+\frac{\dot{m}}{C \rho}\right)$

$+\frac{\dot{m}}{\rho}\left(\dot{R}+\frac{\dot{m}}{2 \rho}+\frac{\dot{R} \dot{m}}{2 c \rho}\right)+\left(1-\frac{\dot{R}}{c}\right) \frac{R}{c \rho} \frac{d P_{B}}{d t}$

where $d / d_{0}$ is the time derivative; $c$ is the sound velocity in liquid; $\rho$ is the liquid density; $\dot{m}$ is the mass exchange rate of the bubble per unit area and unit time; $P_{B}$ is the fluid pressure of the outer boundary of the bubble; $P_{S}$ is a nonparametric driving pressure; $P_{0}$ is the static liquid pressure.

Equation (1) does not consider the effect of the internal and external boundary pressure gradient at a sufficiently fast bubble wall velocity. Hence, it is necessary to add the lateral pressure gradient of the bubble wall $(1-\dot{R} / c)$. The additional factor can weaken the collapse strength of the bubble especially under the high bubble wall velocity.

$P_{B}(t)$ is closely related to [8]:

$P_{B}(t)=P_{g}(t)-\frac{2 \sigma}{R}-\frac{4 \mu}{R}\left(\dot{R}-\frac{\dot{m}}{\rho}\right)-\dot{m}^{2}\left(\frac{1}{\rho}-\frac{1}{\rho_{g}}\right)$

where $\sigma$ is the surface tension; $P_{g}$ is the gas density inside the bubble.

$P_{g}(t)$ can be calculated by Van der Waals equation:

$\left(P_{g}(t)+a / v^{2}\right)(v-b)=R_{g} T$

where $R_{g}$ is the ideal gas parameter; $T$ is the temperature inside the bubble; $v$ is the molar volume; $a$ and $b$ are Var der Waals parameters.

The temperature inside the bubble $(T)$ can be obtained as follows [10]:

$E=\frac{n_{a i r}}{n_{t}} C_{v, \text { air }} T+\frac{n_{H_{2} O}}{n_{t}} C_{V, H_{2} O} T-\left(\frac{n_{t}}{N_{A}}\right)^{2} \frac{a}{V}$

where $n$ is the number of a certain molecule in the bubble; the subscript of $\mathrm{n}$ is the name of the molecule; $n_{t}$ is the total number of all molecules in the bubble; $E$ is the internal energy of the bubble; $C_{\text {air }}\left(C_{H 2 O}\right)$ is the equivalent molar heat capacity of the air/vapor; $N A$ is the Avogadro constant; $V$ is the volume of the bubble.

Let $\Delta E$ be the variation in the internal energy of the bubble over the time $\Delta t$ :

$\Delta E(t)=-P_{g(t)} \Delta V(t)+4 \pi R^{2} \dot{m} e_{H_{2} 0} \Delta_{t}+\left.4 \pi R^{2} \kappa \frac{\partial T}{\partial r}\right|_{r=R} \Delta t$

where $\Delta V$ is the variation in the volume of the bubble over $\Delta t$; $e_{H 2 O}$ is the energy of water evaporation or vapor condensation; $M$ is the total mass of molecules in the bubble; $\kappa$ is the heat transfer coefficient; $r$ is the distance from the bubble centre. Among them, the evaporation or condensation energy can be expressed as:

$e_{H_{2} O}=\frac{C_{V, H_{2} O}}{N_{A}} T_{B}$

Thus,

$\left.\frac{\partial T}{\partial r}\right|_{r=R}=\frac{T_{B}-T}{n \lambda}$

where $T_{B}$ is temperature outside the bubble; $n \lambda \mathrm{s}$ thermal boundary layer of the bubble; $n$ is a constant; $\lambda$ is the mean free path of a gas particle.

Let $\dot{m}$ be the net velocity of water evaporation or vapor condensation per unit area and unit time [11]:

$\dot{m}=\frac{\alpha_{M}}{\left(2 \pi R_{V}\right)^{1 / 2}}\left(\frac{P_{V}^{*}}{T_{0}^{1 / 2}}-\frac{\Gamma P_{V}}{T^{1 / 2}}\right)$

where $\alpha_{M}$ is the self-adjusting coefficient of water evaporation and vapor condensation (the coefficient is assumed as constant); $R_{V}$ is the parameter of the constant vapor; $P_{V}^{*}$ is the pressure of saturated vapor inside the bubble; $T_{0}$ is the liquid temperature; $T$ is the temperature inside the bubble; $P_{V}$ is the partial pressure of vapor in the bubble. Among them, $P_{V}$ can be obtained by the equation below.

$P_{v}=\frac{n_{H_{2} O}}{n_{t}} P_{g}$

The coefficient can be expressed as:

$\Gamma=\exp \left(-\Omega^{2}\right)-\Omega \sqrt{\pi}\left(1-\frac{2}{\sqrt{\pi}} \int_{0}^{\Omega} \exp \left(-x^{2}\right) d x\right)$

where

$\Omega=\frac{\dot{m}}{P_{v}}\left(\frac{R_{v} T}{2}\right)^{1 / 2}$

The number of vapor molecules in the bubble is:

$n_{\mathrm{H}_{2} \mathrm{O}}(t+\Delta t)=n_{\mathrm{H}_{2} \mathrm{O}}(t)+\frac{N_{A}}{M_{\mathrm{H}_{2} \mathrm{O}}} 4 \pi R^{2} \dot{m} \Delta t$

\section{NUMERICAL SIMULATION OF ULTRASONIC CAVITATION BUBBLE}

The initial conditions at time $t=0$ were set as follows: $R=R_{0}, \dot{R}=0, T=T_{0}, \dot{P}_{B}=0, \dot{P}_{g}=0, \quad P_{S}=0, \dot{m}=0, \ddot{m}=0$. Besides, the initial bubble radius $R_{0}=6 \mu \mathrm{m}$, ultrasonic frequency $f=25.6 \mathrm{kHz}$, ultrasonic wave amplitude $P_{a}=1.5 P_{0}$, with $P_{0}$ being the ambient liquid pressure. The dynamic 
motion of the bubble was simulated in Matlab. The simulation results are illustrated in Figures 1 4.

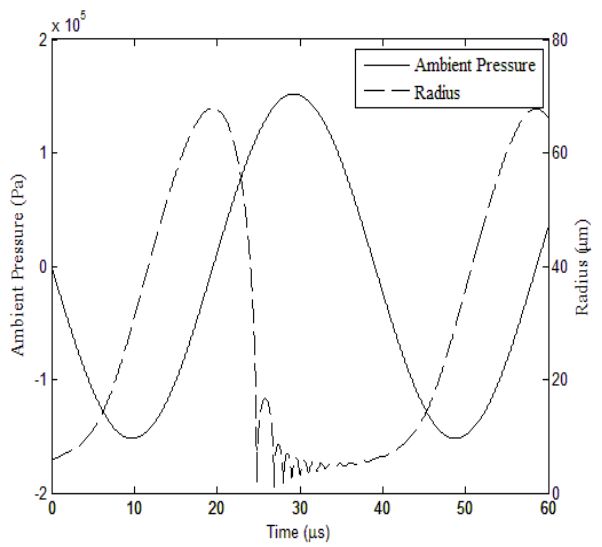

Figure 1. The time-varying patterns of ultrasonic pressure and bubble radius

Figure 1 shows the variation in ultrasonic pressure and bubble radius over the time. The longitudinal axes on the left and the right are the pressure of driving ultrasonic wave and the radius of the cavitation bubble, respectively. The ultrasonic pressure curve and the bubble radius curve are represented as solid line and dotted line, respectively. It can be seen that an alternatively positive and negative ultrasonic field was produced when the liquid was subject to sine ultrasonic wave. The bubble expanded under negative pressure, and shrunk, collapsed and rebounded under positive pressure.

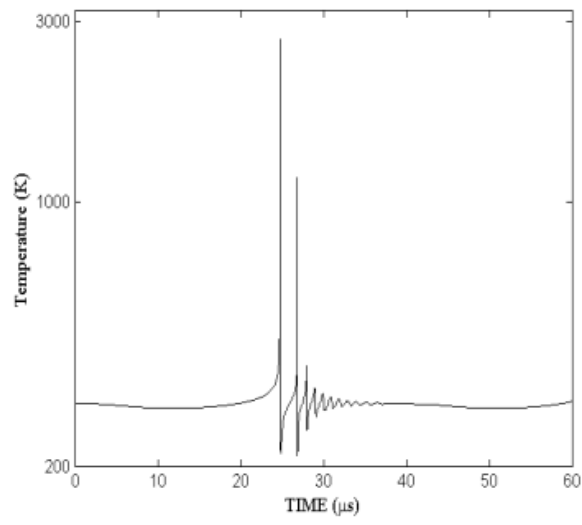

Figure 2. The time-varying pattern of bubble temperature

Figure 2 displays the variation in the bubble temperature over the time. As shown in the figure, the bubble temperature dropped slightly in the expansion phase of the bubble, and increased at a slow pace in the preliminary shrinking phase. Considering the limited change of bubble temperature, these two phases can be considered as an isothermal process. In the later shrinking phase and the collapse phase, the bubble temperature rocketed up, and peaked when the bubble reached the minimum radius.

Figure 3 presents the variation in the bubble pressure over the time. It is clear that the bubble pressure reduced in the expansion phase of the bubble at minimized at $100 \mathrm{~Pa}$. In the preliminary shrinking phase, the bubble radius shrank and the bubble pressure gradually increased. In the later shrinking phase and the collapse phase, the bubble pressure exhibited an upward trend, thanks to the shrinking radius and rapid temperature rise of the bubble. The maximum bubble pressure reached $10^{9} \mathrm{~Pa}$.

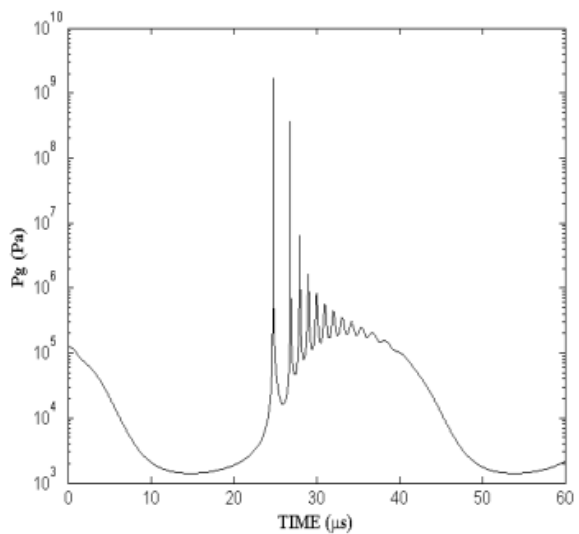

Figure 3. The time-varying pattern of bubble pressure

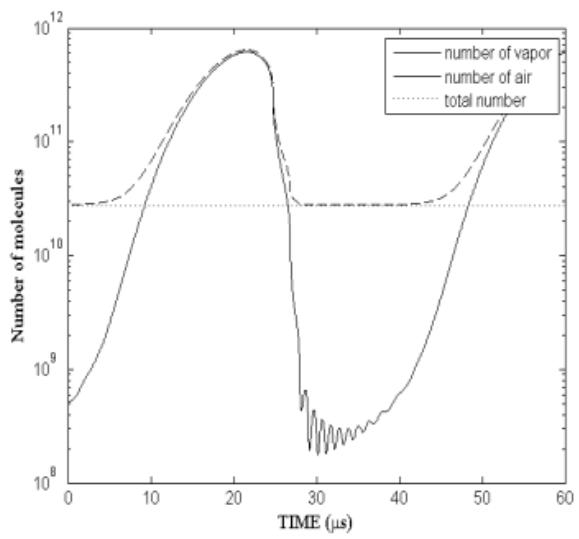

Figure 4. The time-varying patterns of molecules in the bubble

Figure 4 depicts the variation in the number of molecules in the bubble. The number of vapor molecules, the number of gas molecules, and the number of all molecules are represented in the solid line, the dash line and the dotted line, respectively. As shown in the figure, a large number of vapor molecules entered the bubble through the bubble wall in the expansion phase and the initial shrinking phase. In the later shrinking phase and the collapse phase, lots of vapor molecules condensed (the rate of condensation was faster than the rate of evaporation), leading to a plunge in the number of vapor molecules in the bubble. Overall, the maximum number of vapor molecules in the bubble is 1,000 times the initial number of vapor molecules.

\section{EFFECTS OF DIFFERENT FACTORS ON BUBBLE MOTION}

This section aims to disclose the effects of multiple factors on the motion of ultrasonic cavitation bubble. In actual practice, the ultrasonic cavitation happens in different environments, and varies with the external factors. In light of this, the author explored how the initial radius, ultrasonic frequency and ultrasonic amplitude influence the bubble motion. 


\subsection{The cavitation bubble motion as a function of time under different initial radius}

To reveal the effect of the initial radius on bubble motion, the initial radius was set to $2.5 \mu \mathrm{m}, 4.5 \mu \mathrm{m}$ and $6.5 \mu \mathrm{m}$, respectively, and all the other factors were kept constant.

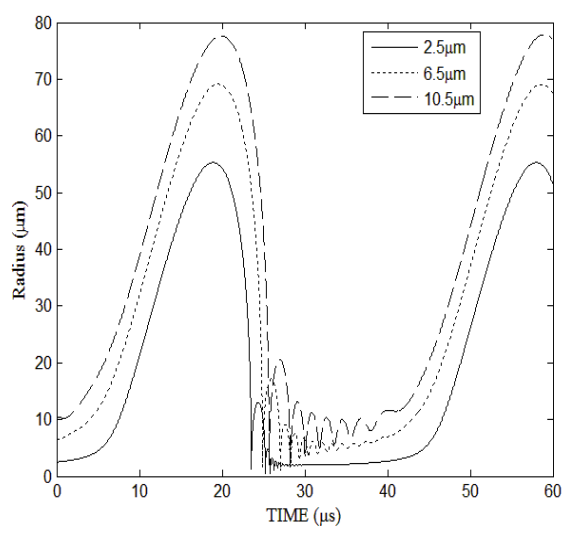

Figure 5. The time-varying patterns of bubble radius with different initial radiuses

Figure 5 describes the variation in bubble radius with different initial radiuses over the time. It can be inferred that the initial radius is positively correlated with the maximum bubble radius, and the energy absorbed by the bubble under negative pressure. Therefore, the bubble with a large initial radius takes a long time to reach the shrinking phase and the first minimum value.

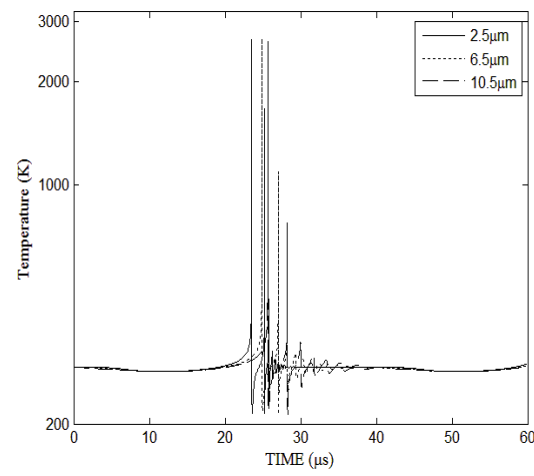

Figure 6. The time-varying patterns of bubble temperature with different initial radiuses

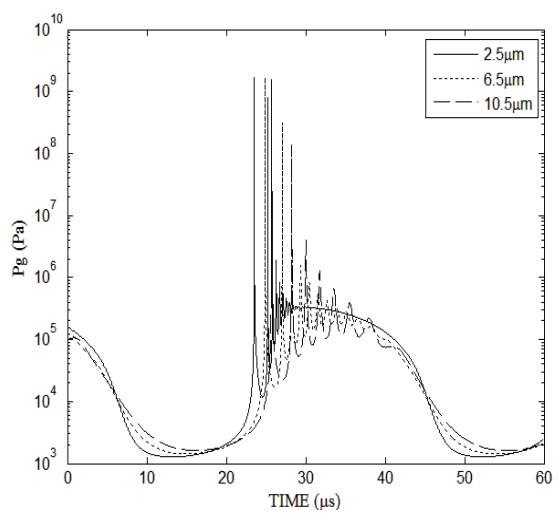

Figure 7. The time-varying patterns of bubble pressure with different initial radiuses
Figures 6 and 7 respectively display the variation in bubble temperature and bubble pressure over the time. It is observed that the bubble temperature and bubble pressure reached the maximum values early if the bubble had a small radius. Besides, the maximum temperature and pressure decreased, but only slightly, if the bubble had a large initial radius. In general, the initial bubble radius has little to do with the temperature and pressure inside the bubble.

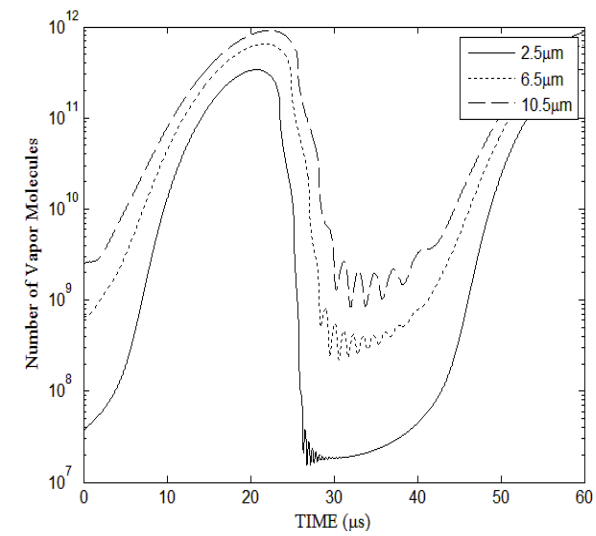

Figure 8. The time-varying patterns of the number of vapor molecules with different initial radiuses

Figure 8 shows the variation in the number of vapor molecules over the time. It can be seen that the initial radius is positively correlated with the number of vapor molecules in the bubble. The positive correlation is attributed to the dependence of the number of vapor molecules on the bubble pressure. The lower the pressure, the greater the number of vapor molecules.

\subsection{Effect of ultrasonic frequency on bubble motion}

Without changing the other factors, different ultrasonic frequencies were selected (i.e. $20 \mathrm{kHz}, 33.3 \mathrm{kHz}$ and $45 \mathrm{kHz}$ ) to simulate the effect of ultrasonic frequency on the motion of ultrasonic cavitation bubble.

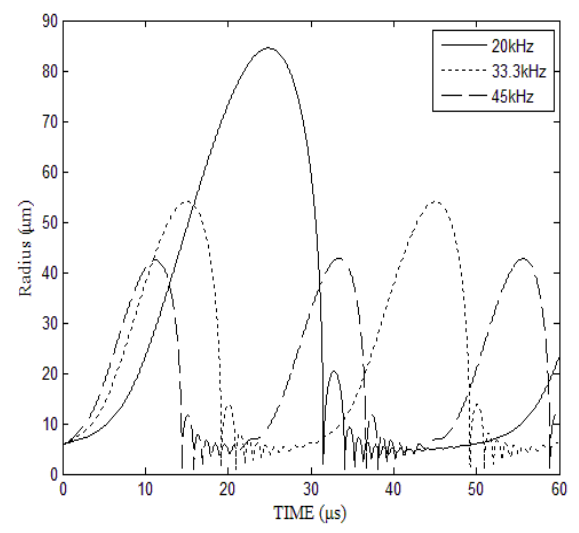

Figure 9. The time-varying patterns of bubble radius with different ultrasonic frequencies

Figure 9 presents the variation in bubble radius over the time at different ultrasonic frequencies. It is clear that the ultrasonic frequency had a prominent impact on the bubble radius. The smaller the frequency, the larger the radius of the bubble, especially the maximum radius. This is because the ultrasonic wave period is negatively correlated with the 
ultrasonic frequency. In addition, the bubble absorbed more energy under the negative pressure when the positive and negative pressure phases of the ultrasonic wave became longer, resulting in a larger bubble radius.

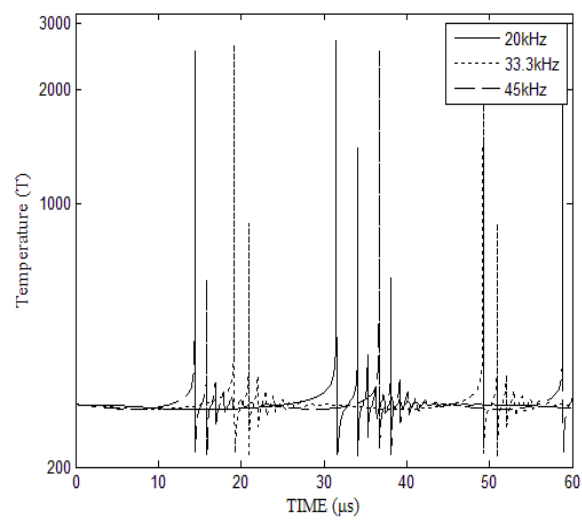

Figure 10. The time-varying patterns of bubble temperature with different ultrasonic frequencies

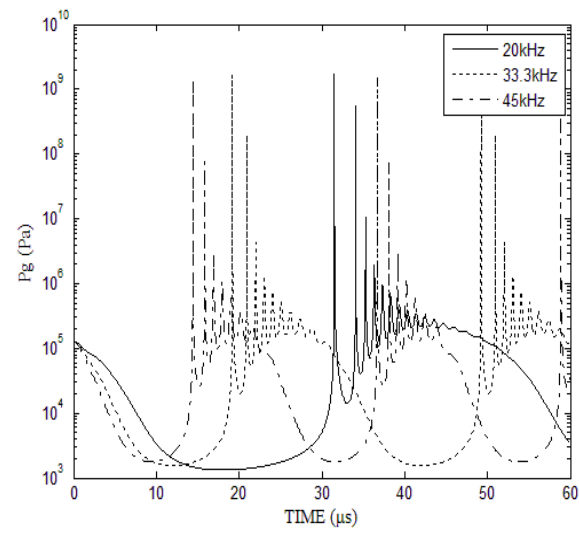

Figure 11. The time-varying patterns of bubble pressure with different ultrasonic frequencies

Figures 10 and 11 respectively illustrate the variation in bubble temperature and bubble pressure over the time. With the increase in the ultrasonic frequency, the peak temperature and the peak pressure slightly decreased. This means the ultrasonic frequency has a little impact on the bubble temperature and bubble pressure.

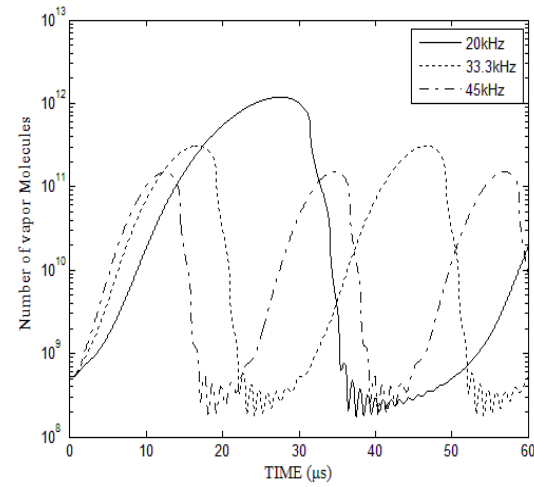

Figure 12. The time-varying patterns of the number of vapor molecules with different ultrasonic frequencies
Figure 12 shows the variation in the number of vapor molecules over the time. As the ultrasonic frequency increased, the number of vapor molecules in the bubble exhibited a decreasing trend. The phenomenon can be explained as follows. The net evaporation rate mainly relies on bubble temperature and bubble pressure. The lower the pressure, the greater the increment of the global number of vapor molecules. When the ultrasonic frequency grew, the negative pressure phase was shortened, and so it was with the low-pressure period inside the bubble. In this case, fewer and fewer vapor molecules would accumulate in the bubble.

\subsection{Effect of ultrasonic amplitude on bubble motion}

Without changing the other factors, several ultrasonic amplitudes were selected (i.e. $1.25 P_{0}, 2 P_{0}$ and $2.75 P_{0}$ ) to simulate the effect of ultrasonic amplitude on the motion of ultrasonic cavitation bubble.

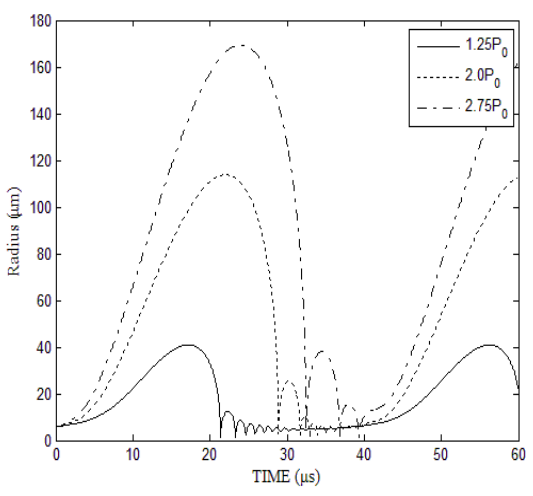

Figure 13. The time-varying patterns of bubble radius with different ultrasonic amplitudes

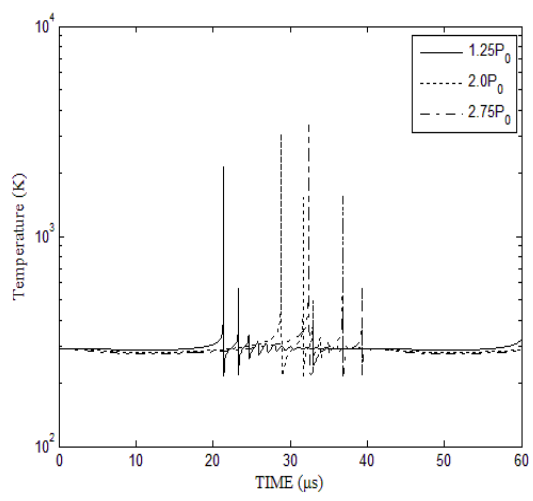

Figure 14. The time-varying patterns of bubble temperature with different ultrasonic amplitudes

Figure 13 presents the variation in bubble radius over the time at different ultrasonic amplitudes. It can be seen that the ultrasonic amplitude had a prominent impact on the bubble radius. The amplitude is positively correlated with the bubble radius, especially the maximum radius. The positive correlation can be explained as follows. When the ultrasonic frequency remains the same, the increase in the ultrasonic amplitude means more energy is absorbed by the bubble under the negative pressure. With a profound amount of energy, the bubble will expand in radius, which is exhibited as a long expansion phase.

Figures 14 and 15 respectively illustrate the variation in bubble temperature and bubble pressure over the time. With 
the increase in the ultrasonic amplitude, the temperature and pressure curves both had a higher peak. The growth in temperature and pressure at the collapse is attributable to the increase in the energy absorbed by the bubble under negative pressure when the ultrasonic amplitude widens.

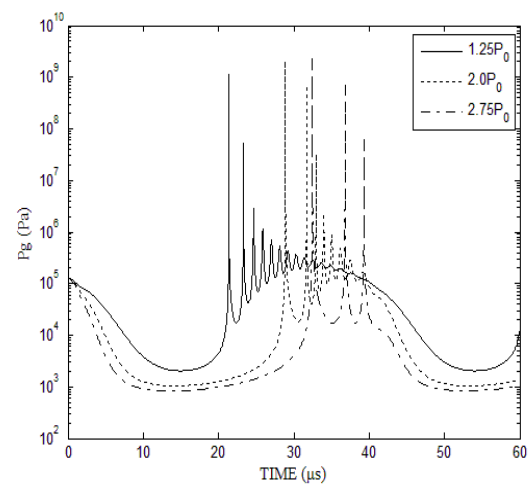

Figure 15. The time-varying patterns of bubble pressure with different ultrasonic amplitudes

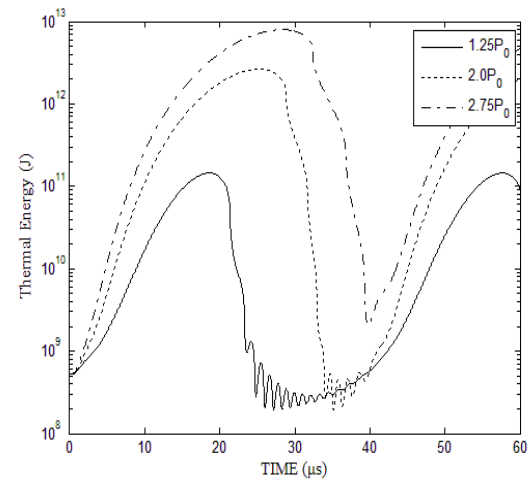

Figure 16. The time-varying patterns of the number of vapor molecules with different ultrasonic amplitudes

Figure 16 shows the variation in the number of vapor molecules over the time. As the ultrasonic frequency increased, the number of vapor molecules in the bubble was on the rise. The trend can be explained as follows. The net evaporation rate mainly relies on bubble temperature and bubble pressure. The greater the ultrasonic amplitude, the lower the bubble in the expansion phase. Under a high net evaporation rate increases, the ultrasonic amplitude and the bubble radius will be relatively large, pushing up the number of vapor molecules in the bubble.

\section{CONCLUSION}

This paper simulates the ultrasonic cavitation on Matlab considering the water evaporation and vapor condensation. Various influencing factors were introduced to analyse the bubble motion, including initial radius, ultrasonic frequency and ultrasonic amplitude. The motion of the bubble was characterized by such parameters as radius, temperature, pressure, internal energy and the number of vapor molecules. Through the simulation, it is discovered that the change of the initial radius had a little impact on the bubble temperature and bubble pressure. However, the radius variation exerted an obvious influence on the other motion parameters. These parameters shared a similar change pattern. For ultrasonic frequency, the change in frequency had a limited effect on the bubble temperature and bubble pressure, but a significant impact on the other motion parameters. These parameters varied in different ranges. In addition, the ultrasonic amplitude had a rather prominent impact on all motion parameters of the cavitation bubble.

\section{ACKNOWLEDGMENT}

This study was jointly supported by the Fundamental Research Funds for the Central Universities of China (No. N150304001, No. N160304003).

\section{REFERENCES}

[1] Du X.M., Dang Z., Zhang Z.F., Bai B.F. (2011). Hydrogen production from sonolysis of aqueous methanol solution, CIESC Journal, Vol. 62, No. 6, pp. 1669-1674. DOI: $\underline{10.3969 / j . i s s n .0438-}$ 1157.2011.06.029

[2] Plesset M.S., Hsieh D. (1960). Theory of gas bubble dynamics in oscillating pressure fields, Physics of Fluids, Vol. 3, No. 6, pp. 882-892. DOI: 10.1063/1.1706152

[3] Noltingk B.E., Neppiras E.A. (1950). Cavitation produced by ultrasonics, Proceedings of the Physical Society. Section B, Vol. 63, No. 9, pp. 674-685.

[4] Gilmore F.R. (1952). The growth or collapse of a spherical bubble in a viscous compressible liquid, California Institute of Technology, Pasadena, USA, p. 561.

[5] An Y., Ying C.F. (2005). Model of single bubble sonoluminescence, Physical Review E Statistical Nonlinear \& Soft Matter Physics, Vol. 71, No. 3, p. 036308. DOI: 10.1103/PhysRevE.71.036308

[6] Wang P.H. (2003). The influencing factors of ultrasonic cavitation, Journal of Hebei Institute of Technology, Vol. 25, No. 4, pp. 154-161. DOI: 10.3969/j.issn.1674-0262.2003.04.029

[7] Xu W.L., He Y.F., Wang Y.Q. (2005). Solution and process simulation of ultrasonic cavitation bubble motion equation, Journal of Yangzhou University (Natural Science Edition), Vol. 8, No. 1, pp. 55-59, 68. DOI: $\underline{10.3969 / \mathrm{j} . i s s n .1007-824 X .2005 .01 .014}$

[8] Yasui K. (1996). Variation of Liquid temperature at bubble wall near the Sonoluminescence Threshold, Journal of the Physical Society of Japan, Vol. 65, No. 9, pp. 2830-2840. DOI: 10.1143/JPSJ.65.2830

[9] Yasui K. (1995). Effects of thermal conduction on bubble dynamics near the sonolumiescence Threshold, Journal of the Acoustical Society of America, Vol. 98, No. 5, pp. 2772-2782. DOI: 10.1121/1.413242

[10] Yasui K. (1997). Alternative model of single-bubble sonoluminecence, Physical Review E, Vol. 56, No. 6, pp. 6750-6760. DOI: $10.1103 /$ PhysRevE.56.6750

[11] Shen Y., Yasui K., Sun Z.H., Mei B., You M.Y., Zhu T. (2016). Study on the spatial distribution of the liquid temperature near a cavitation bubble wall, Ultrasonics Sonochemistry, Vol. 29, pp. 394-400. DOI: $10.1016 /$ j.ultsonch.2015.10.015 